How to cite: Giurgiu, D.-A., Tudorache, A.-V., Mirea, A. (2021) A Blizzard Episode in Oltenia (S-W Romania). 2021 "Air and Water - Components of the Environment" Conference Proceedings, Cluj-Napoca, Romania, p. 920, DOI: 10.24193/AWC2021_02.

\title{
A BLIZZARD EPISODE IN OLTENIA (S-W ROMANIA)
}

\author{
Diana-Alexandra GIURGIU ${ }^{1}$, Andreea-Violeta TUDORACHE ${ }^{1,3}$, Adrian \\ MIREA ${ }^{\text {b2 }}$ \\ DOI: 10.24193/AWC2021_02
}

\begin{abstract}
Blizzard phenomena is a complex atmospheric event, in which the snow that falls or has fallen is blown away by the wind, so that the visibility decrease significant enough and the assessment of the simultaneous snow fall is impossible (Țisslea, D. et al. 1965). The region of Oltenia is featured by a climate with Mediterranean influences, with the dominance of northwest and east winds (Roșu, Al. Et al. 1971). The contact between the cold and the warm air masses leads to the increase of the horizontal baric gradient, which causes strong wind intensification. Due to the geographical position of the Carpathian chain, the penetration of colder and denser air is directed towards the east and the south of Romania, thereby has an impact on the study area (***Climate of Romania 2008). Out of all the natural phenomena during the cold season, blizzard is by far the most versatile, with a heavily impact on society and environment (Teodoreanu 2004). The purpose of this paper is to emphasize the hydrometeorological outcome of the 2018 blizzard episode from February $24^{\text {th }}$ to March $2^{\text {nd }}$. In order to achieve this, we will identify the spatial and temporal variability of the hazard in the region of Oltenia, the atmospheric mechanism of this late blizzard occurrence, its climatic characteristics and the consequences on the rivers runoff regime. We will analyze the synoptic conditions under which the blizzard appeared, the recorded meteorological data and a series of hydrological data from the stations located on the main rivers (Jiu, Olt and Danube) in order to determine the synoptic weather pattern, the successive number of days with blizzard, the dominant direction and the average and maximum wind speed, the average and maximum amount of precipitation, as well as for the thickness of snow layer and the daily liquid levels and flows.
\end{abstract}

Keywords: Oltenia, blizzard, precipitation, snow

\section{THE SYNOPTIC CONDITIONS}

The 2018 Oltenia blizzard episode from February $24^{\text {th }}$ to March $2^{\text {nd }}$ was a typical meteorological phenomenon for this region and had its maximum intensity in this area on February $26^{\text {th }}$ and $27^{\text {th }}$.

\footnotetext{
${ }^{1}$ University of Bucharest - Faculty of Geography - Doctoral School "Simion Mehedinți-Nature and Sustainable Development”, e-mail: g.dianalex@gmail.com

${ }^{2}$ Romanian National Meteorological Administration, e-mail: tudoracheav@gmail.com

${ }^{3}$ National Institute of Hydrology and Water Management, e-mail: adymirea@gmail.com
} 
The weather was colder than usual for the end of the second month of the year, as from a few days before the blizzard. This situation happened due to the ingress of a cold air mass of continental polar origin (Fig. 1), related to a wide extension over Central and Eastern Europe, therefore over Romania too, of the Scandinavian Anticyclone. The presence of this high-pressure area over Romanian territory represented the first necessary ingredient for the blizzard phenomenon to manufacture.

A
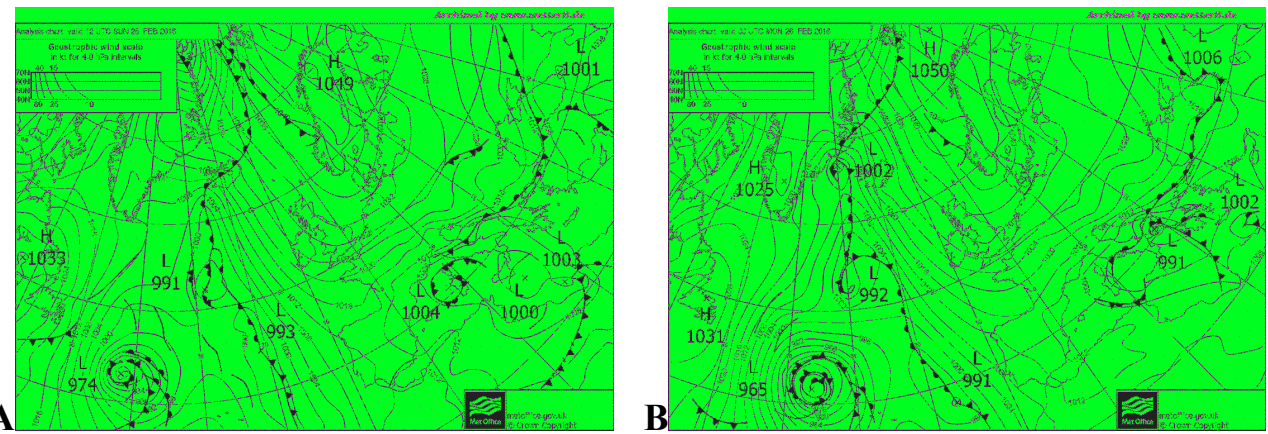

Fig. 1. Synoptic context at ground level over Europe for 2018 February $25^{\text {th }}$ (A) and $26^{\text {th }}$ (B) (source: http://www1.wetter3.de/Archiv))

The Omega type blockage (Fig. 2) favored the large expansion in time and space of the cold air mass, which initially due to the low moisture intake did not cause significant precipitation. This blocking circulation has the potential to intensify the phenomena up to the threshold of severe weather.

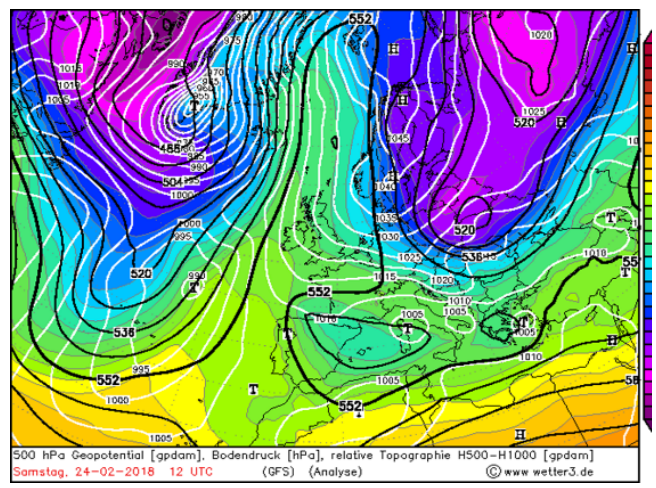

Fig. 2. Geopotential at $500 \mathrm{hPa}$ level (black lines), ground pressure (white lines) and geopotential difference between 500 and $1000 \mathrm{hPa}$ levels over Europe for 2018 February $24^{\text {th }}$ (source: http://www1.wetter3.de/Archiv/)

The phenomena, namely: heavy snowfall and wind gusts, have become more frequent since February $26^{\text {th }}$ morning and during most of that day. This development was possible due to the coupling between the anticyclone already established over our country and the Mediterranean cyclone arisen in the eastern basin of the Mediterranean Sea, that traveled on a classical trajectory to the Black Sea basin, gradually crossing the southern half of our territory (Fig. 3). 


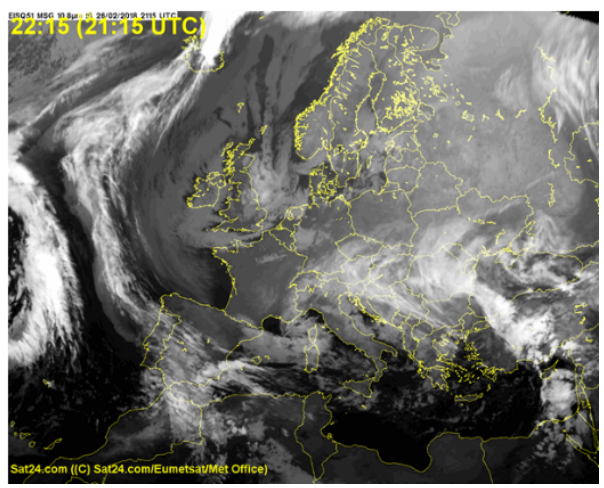

Fig. 3. Satellite image over Europe for 2018 February $26^{\text {th }}$, 21:15 UTC (source: www.wetterzentrale.de)

The organization of these cyclones is the second needed ingredient for the blizzard occurrence. They are responsible for the advection of warmer and humid air masses (Fig. 5.) that entail heavy rainfall on their route up to the moment of occlusion.
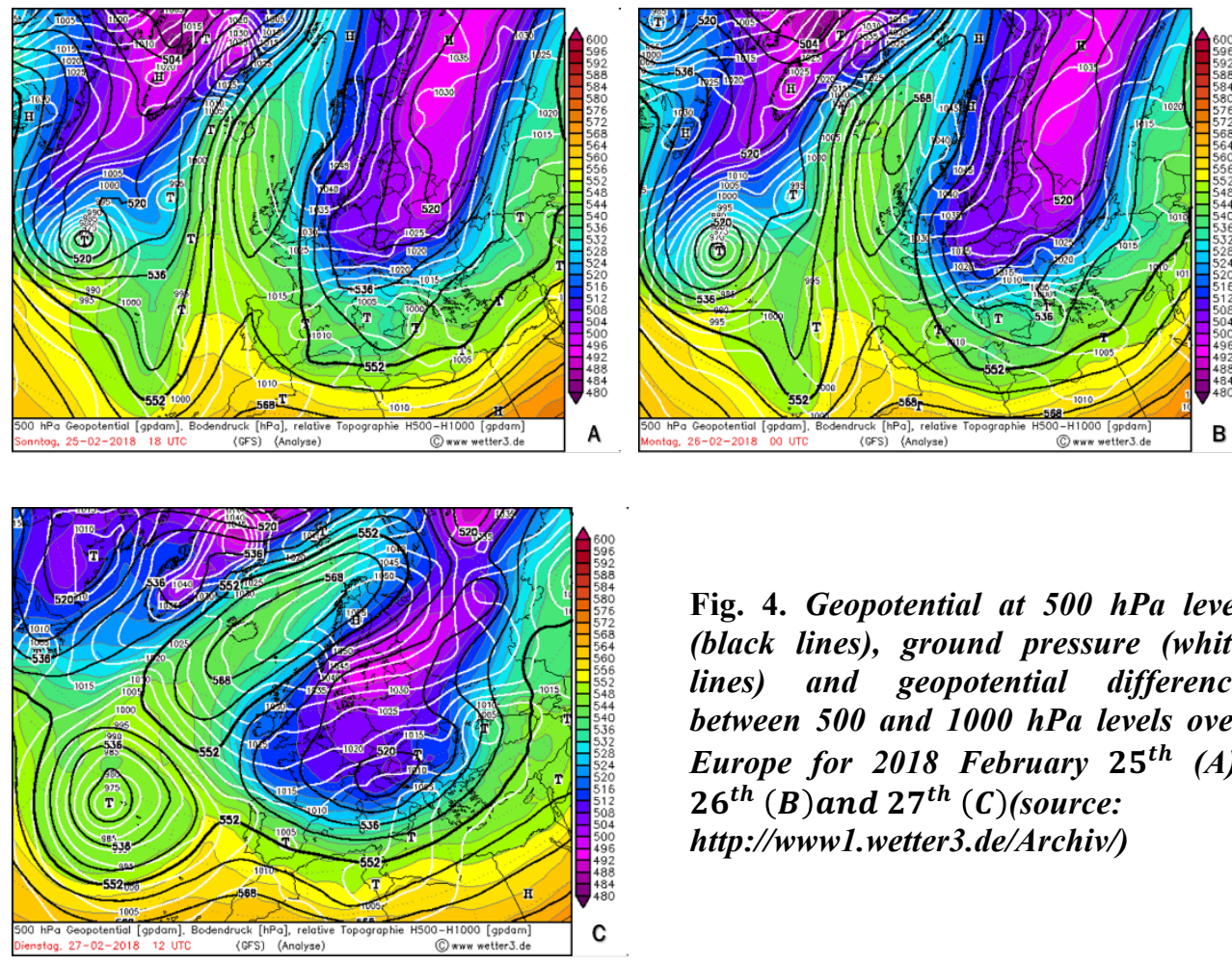

Fig. 4. Geopotential at 500 hPa level (black lines), ground pressure (white lines) and geopotential difference between 500 and 1000 hPa levels over Europe for 2018 February $25^{\text {th }}(A)$, $26^{\text {th }}(B)$ and $27^{\text {th }}(C)$ (source: http://www1.wetter3.de/Archiv/)

According to the atmospheric soundings below it can easily be observed the following: the existent cold air mass close to the surface; wind direction dominant from the east near the surface, fitting the specific trajectory of the cyclone, parallel 
with the west-south-west direction in the upper atmosphere; the humid air intrusion from around $800 \mathrm{~m}$ altitude $(900 \mathrm{hPa}$ level $)$.
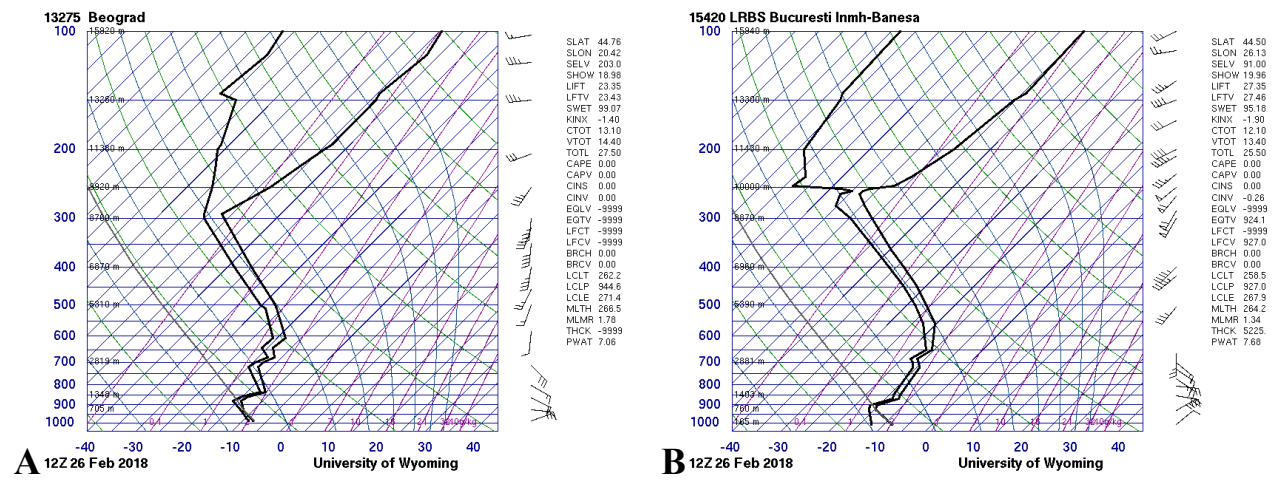

Fig. 5. Atmospheric soundings for 2018 February $26^{\text {th }}, 12$ UTC in Belgrade (A) and Bucharest (B) (source: http://weather.uwyo.edu/upperair/sounding.html)

According to the classification of the seven baric types that favor the development of blizzards in Romania, this episode can be classified as type I, which involves the existence of an anticyclonic belt in the north or northeast of Romania and the evolution of a depression baric field in the Mediterranean basin. This is also the most frequent and in the same time specific baric pattern of blizzard appearance in our country.

\section{METEOROLOGICAL DATA}

The recorded meteorological data used in our analysis, were the hourly values for the dominant wind direction, wind speed and snow layer thickness and the three hours precipitation amount.

We have taken under consideration four relevant meteorological stations from Oltenia area: Craiova, Slatina, Caracal and Drăgășani. In the tables below it can be observed the evolution during February $26^{\text {th }}$ and $27^{\text {th }}$, when the event was at its maximum intensity.

In Table 1 for Craiova meteorological station, shortly after begging of the blizzard the snow layer was $7 \mathrm{~cm}$ and by the end of the event touched $46 \mathrm{~cm}$. Wind direction during these two days was mostly east, east-northeast and the wind speed reached maximum in the evening of February $26^{\text {th }}$.

In Table 2 for Slatina meteorological station, when the blizzard intensified the snow layer was $6 \mathrm{~cm}$ and the second day reached $50 \mathrm{~cm}$. Wind direction during these two days was from east sector and the wind speed maximum was at the beginning of the event. 
Table 1. Meteorological data for Craiova meteorological station for February $26^{\text {th }}$ and $27^{\text {th }}$ (source: Romanian National Meteorological Administration)

\begin{tabular}{|c|c|c|c|c|c|}
\hline \multicolumn{5}{|c|}{ Hourly weather data - Craiova meteorological station } \\
\hline & & \multicolumn{2}{|c|}{ Wind } & $\begin{array}{c}\text { Precipitation } \\
\text { for } 3 \text { hours }\end{array}$ & $\begin{array}{c}\text { Layer of } \\
\text { snow }\end{array}$ \\
\hline Date & Hour & Direction $\left({ }^{\circ}\right)$ & Speed $(\mathrm{m} / \mathrm{s})$ & $(\mathrm{mm})$ & $(\mathrm{cm})$ \\
\hline $2 / 26 / 2018$ & 00 & ENE & 5.5 & 0.7 & 7 \\
\hline $2 / 26 / 2018$ & 01 & ENE & 8.2 & & \\
\hline $2 / 26 / 2018$ & 02 & ENE & 6.8 & & \\
\hline $2 / 26 / 2018$ & 03 & E & 5.2 & 1.0 & 7 \\
\hline $2 / 26 / 2018$ & 04 & ENE & 5.5 & & \\
\hline $2 / 26 / 2018$ & 05 & ENE & 8.8 & & \\
\hline $2 / 26 / 2018$ & 06 & ENE & 6.3 & 0.8 & 7 \\
\hline $2 / 26 / 2018$ & 07 & E & 6.8 & & \\
\hline $2 / 26 / 2018$ & 08 & $E$ & 9.4 & & \\
\hline $2 / 26 / 2018$ & 09 & ENE & 7.3 & 1.7 & 10 \\
\hline $2 / 26 / 2018$ & 10 & ENE & 5.9 & & 11 \\
\hline $2 / 26 / 2018$ & 11 & ENE & 7 & & 13 \\
\hline $2 / 26 / 2018$ & 12 & ENE & 6.9 & 2.8 & 15 \\
\hline $2 / 26 / 2018$ & 13 & E & 7.3 & & 15 \\
\hline $2 / 26 / 2018$ & 14 & E & 6.6 & & 17 \\
\hline $2 / 26 / 2018$ & 15 & E & 7.3 & 2.2 & 19 \\
\hline $2 / 26 / 2018$ & 16 & E & 6.3 & & 21 \\
\hline $2 / 26 / 2018$ & 17 & E & 8 & & 21 \\
\hline $2 / 26 / 2018$ & 18 & E & 7.6 & 1.0 & 22 \\
\hline $2 / 26 / 2018$ & 19 & E & 10.1 & & 23 \\
\hline $2 / 26 / 2018$ & 20 & E & 7.1 & & 26 \\
\hline $2 / 26 / 2018$ & 21 & ESE & 5.8 & 1.4 & 29 \\
\hline $2 / 26 / 2018$ & 22 & E & 6.4 & & 30 \\
\hline $2 / 26 / 2018$ & 23 & ESE & 4.7 & & 32 \\
\hline & & & & & \\
\hline
\end{tabular}

\begin{tabular}{|c|c|c|c|c|c|}
\hline \multicolumn{5}{|c|}{ Hourly weather data - Craiova meteorological station } \\
\hline & & \multicolumn{2}{|c|}{ Wind } & $\begin{array}{c}\text { Precipitation } \\
\text { for } 3 \text { hours }\end{array}$ & $\begin{array}{c}\text { Layer of } \\
\text { snow }\end{array}$ \\
\hline Date & Hour & Direction $\left(^{\circ}\right)$ & Speed $(\mathrm{m} / \mathrm{s})$ & $(\mathrm{mm})$ & $(\mathrm{cm})$ \\
\hline $2 / 27 / 2018$ & 00 & ESE & 5.9 & 2.6 & 34 \\
\hline $2 / 27 / 2018$ & 01 & ESE & 3.4 & & 36 \\
\hline $2 / 27 / 2018$ & 02 & ESE & 2.8 & & 38 \\
\hline $2 / 27 / 2018$ & 03 & SE & 2.3 & 1.6 & 41 \\
\hline $2 / 27 / 2018$ & 04 & ESE & 2.5 & & 43 \\
\hline $2 / 27 / 2018$ & 05 & ESE & 2.8 & & 44 \\
\hline $2 / 27 / 2018$ & 06 & ESE & 3.1 & 1.2 & 44 \\
\hline $2 / 27 / 2018$ & 07 & SSE & 2.5 & & 46 \\
\hline $2 / 27 / 2018$ & 08 & S & 1.4 & & 49 \\
\hline $2 / 27 / 2018$ & 09 & SE & 0.7 & 1.8 & 50 \\
\hline $2 / 27 / 2018$ & 10 & $E$ & 1.8 & & 50 \\
\hline $2 / 27 / 2018$ & 11 & ESE & 2 & & 50 \\
\hline $2 / 27 / 2018$ & 12 & SE & 1.9 & 0.5 & 50 \\
\hline $2 / 27 / 2018$ & 13 & ESE & 1.9 & & 48 \\
\hline $2 / 27 / 2018$ & 14 & ESE & 2.4 & & 48 \\
\hline $2 / 27 / 2018$ & 15 & ESE & 2.3 & 0.0 & 47 \\
\hline $2 / 27 / 2018$ & 16 & $\mathrm{E}$ & 2.4 & & 46 \\
\hline $2 / 27 / 2018$ & 17 & ESE & 3.1 & & 46 \\
\hline $2 / 27 / 2018$ & 18 & $\mathrm{E}$ & 2.7 & 0.0 & 46 \\
\hline $2 / 27 / 2018$ & 19 & $\mathrm{ENE}$ & 2.2 & & \\
\hline $2 / 27 / 2018$ & 20 & $\mathrm{E}$ & 4.1 & & \\
\hline $2 / 27 / 2018$ & 21 & $\mathrm{E}$ & 5.1 & 0.1 & 46 \\
\hline $2 / 27 / 2018$ & 22 & $\mathrm{E}$ & 5.6 & & \\
\hline $2 / 27 / 2018$ & 23 & $\mathrm{E}$ & 3.3 & & \\
\hline
\end{tabular}

Table 2. Meteorological data for Slatina meteorological station for February $26^{\text {th }}$ and $27^{\text {th }}$ (source: Romanian National Meteorological Administration)

\begin{tabular}{|c|c|c|c|c|c|}
\hline \multicolumn{5}{|c|}{ Hourly weather data - Slatina meteorological station } \\
\hline & & \multicolumn{2}{|c|}{ Wind } & $\begin{array}{c}\text { Precipitation } \\
\text { for 3 hours }\end{array}$ & $\begin{array}{c}\text { Layer of } \\
\text { snow }\end{array}$ \\
\hline Date & Hour & Direction $\left(^{\circ}\right)$ & Speed $(\mathrm{m} / \mathrm{s})$ & $(\mathrm{mm})$ & $(\mathrm{cm})$ \\
\hline $2 / 26 / 2018$ & 00 & E & 5.4 & 0.6 & 6 \\
\hline $2 / 26 / 2018$ & 01 & ENE & 5.3 & & \\
\hline $2 / 26 / 2018$ & 02 & ENE & 7.7 & & \\
\hline $2 / 26 / 2018$ & 03 & E & 8.3 & 0.7 & 7 \\
\hline $2 / 26 / 2018$ & 04 & E & 8.5 & & \\
\hline $2 / 26 / 2018$ & 05 & ENE & 7.6 & & \\
\hline $2 / 26 / 2018$ & 06 & ENE & 6.3 & 0.3 & 6 \\
\hline $2 / 26 / 2018$ & 07 & E & 7.3 & & \\
\hline $2 / 26 / 2018$ & 08 & ENE & 7.3 & & \\
\hline $2 / 26 / 2018$ & 09 & ENE & 7.1 & 0.4 & 6 \\
\hline $2 / 26 / 2018$ & 10 & ENE & 7.7 & & 6 \\
\hline $2 / 26 / 2018$ & 11 & ENE & 6.9 & & 8 \\
\hline $2 / 26 / 2018$ & 12 & ENE & 7.3 & 1.7 & 9 \\
\hline $2 / 26 / 2018$ & 13 & ENE & 5.2 & & 9 \\
\hline $2 / 26 / 2018$ & 14 & ENE & 4.9 & & 10 \\
\hline $2 / 26 / 2018$ & 15 & E & 6 & 1.9 & 11 \\
\hline $2 / 26 / 2018$ & 16 & E & 5.4 & & 11 \\
\hline $2 / 26 / 2018$ & 17 & E & 5.4 & & 11 \\
\hline $2 / 26 / 2018$ & 18 & E & 4.7 & 1.4 & 12 \\
\hline $2 / 26 / 2018$ & 19 & E & 3.9 & & 12 \\
\hline $2 / 26 / 2018$ & 20 & E & 5.4 & & 13 \\
\hline $2 / 26 / 2018$ & 21 & E & 4.4 & 1.8 & 14 \\
\hline $2 / 26 / 2018$ & 22 & ENE & 4.6 & & 15 \\
\hline $2 / 26 / 2018$ & 23 & E & 5 & & 16 \\
\hline & & & & & \\
\hline
\end{tabular}

\begin{tabular}{|c|c|c|c|c|c|}
\hline \multicolumn{5}{|c|}{ Wourly weather data - Slatina meteorological station } \\
\hline & & \multicolumn{2}{|c|}{ Wind } & $\begin{array}{c}\text { Precipitation } \\
\text { for } 3 \text { hours }\end{array}$ & $\begin{array}{c}\text { Layer of } \\
\text { snow }\end{array}$ \\
\hline Date & Hour & Direction $\left(^{\circ}\right)$ & Speed $(\mathrm{m} / \mathrm{s})$ & $(\mathrm{mm})$ & $(\mathrm{cm})$ \\
\hline $2 / 27 / 2018$ & 00 & ENE & 3.4 & 2.2 & 17 \\
\hline $2 / 27 / 2018$ & 01 & E & 2.7 & & 18 \\
\hline $2 / 27 / 2018$ & 02 & ESE & 2.7 & & 19 \\
\hline $2 / 27 / 2018$ & 03 & ESE & 2.9 & 1.6 & 20 \\
\hline $2 / 27 / 2018$ & 04 & SE & 2.5 & & 22 \\
\hline $2 / 27 / 2018$ & 05 & SE & 2.9 & & 24 \\
\hline $2 / 27 / 2018$ & 06 & ESE & 2.3 & 1.0 & 25 \\
\hline $2 / 27 / 2018$ & 07 & SE & 2.7 & & 26 \\
\hline $2 / 27 / 2018$ & 08 & SSV & 1.7 & & 28 \\
\hline $2 / 27 / 2018$ & 09 & SSE & 2.3 & 2.0 & 29 \\
\hline $2 / 27 / 2018$ & 10 & S & 2.5 & & 31 \\
\hline $2 / 27 / 2018$ & 11 & SSV & 2.3 & & 32 \\
\hline $2 / 27 / 2018$ & 12 & SV & 1.9 & 1.9 & 33 \\
\hline $2 / 27 / 2018$ & 13 & SSE & 0.7 & & 34 \\
\hline $2 / 27 / 2018$ & 14 & V & 0.8 & & 34 \\
\hline $2 / 27 / 2018$ & 15 & SE & 1.3 & 0.5 & 34 \\
\hline $2 / 27 / 2018$ & 16 & ESE & 1.6 & & 33 \\
\hline $2 / 27 / 2018$ & 17 & SE & 3.1 & & 33 \\
\hline $2 / 27 / 2018$ & 18 & ESE & 2.2 & 0.0 & 32 \\
\hline $2 / 27 / 2018$ & 19 & E & 2.2 & & \\
\hline $2 / 27 / 2018$ & 20 & ESE & 2.4 & & \\
\hline $2 / 27 / 2018$ & 21 & E & 3.2 & 0.2 & \\
\hline $2 / 27 / 2018$ & 22 & ENE & 2.9 & & \\
\hline $2 / 27 / 2018$ & 23 & $\mathrm{E}$ & 4.3 & & \\
\hline
\end{tabular}

In Table 3 for Caracal meteorological station, shortly after begging of the blizzard the snow layer was $11 \mathrm{~cm}$ and the second day reached $35 \mathrm{~cm}$. Wind direction during these two days was also from east sector and the wind speed maximum was on the first day of the high intensity event. 
Table 3. Meteorological data for Caracal meteorological station for February $26^{\text {th }}$ and $27^{\text {th }}$ (source: Romanian National Meteorological Administration)

\begin{tabular}{|c|c|c|c|c|c|}
\hline \multicolumn{5}{|c|}{ Hourly weather data - Caracal meteorological station } \\
\hline & & \multicolumn{2}{|c|}{ Wind } & $\begin{array}{c}\text { Precipitation } \\
\text { for 3 hours }\end{array}$ & $\begin{array}{c}\text { Layer of } \\
\text { snow }\end{array}$ \\
\hline Date & Hour & Direction $\left(^{\circ}\right)$ & Speed $(\mathrm{m} / \mathrm{s})$ & $(\mathrm{mm})$ & $(\mathrm{cm})$ \\
\hline $2 / 26 / 2018$ & 00 & $\mathrm{E}$ & 6.7 & 0.8 & 11 \\
\hline $2 / 26 / 2018$ & 01 & $\mathrm{E}$ & 8.3 & & \\
\hline $2 / 26 / 2018$ & 02 & $\mathrm{E}$ & 8.1 & & \\
\hline $2 / 26 / 2018$ & 03 & $\mathrm{E}$ & 7.5 & 0.8 & 11 \\
\hline $2 / 26 / 2018$ & 04 & $\mathrm{E}$ & 8.2 & & \\
\hline $2 / 26 / 2018$ & 05 & $\mathrm{E}$ & 8.4 & & \\
\hline $2 / 26 / 2018$ & 06 & $\mathrm{E}$ & 7.1 & 0.4 & 12 \\
\hline $2 / 26 / 2018$ & 07 & $\mathrm{E}$ & 7.6 & & \\
\hline $2 / 26 / 2018$ & 08 & $\mathrm{E}$ & 8 & & \\
\hline $2 / 26 / 2018$ & 09 & $\mathrm{E}$ & 8 & 0.4 & 12 \\
\hline $2 / 26 / 2018$ & 10 & $\mathrm{E}$ & 7.3 & & 12 \\
\hline $2 / 26 / 2018$ & 11 & $\mathrm{E}$ & 7.3 & & 13 \\
\hline $2 / 26 / 2018$ & 12 & $\mathrm{E}$ & 6.4 & 1.4 & 14 \\
\hline $2 / 26 / 2018$ & 13 & $\mathrm{E}$ & 6.9 & & 14 \\
\hline $2 / 26 / 2018$ & 14 & $\mathrm{E}$ & 6.3 & & 14 \\
\hline $2 / 26 / 2018$ & 15 & $\mathrm{E}$ & 4.9 & 1 & 15 \\
\hline $2 / 26 / 2018$ & 16 & $\mathrm{E}$ & 6.6 & & 15 \\
\hline $2 / 26 / 2018$ & 17 & $\mathrm{E}$ & 7.7 & & 16 \\
\hline $2 / 26 / 2018$ & 18 & $\mathrm{E}$ & 7.9 & 1.6 & 17 \\
\hline $2 / 26 / 2018$ & 19 & $\mathrm{E}$ & 6.1 & & 17 \\
\hline $2 / 26 / 2018$ & 20 & $\mathrm{E}$ & 5.7 & & 17 \\
\hline $2 / 26 / 2018$ & 21 & $\mathrm{E}$ & 5.4 & 1 & 19 \\
\hline $2 / 26 / 2018$ & 22 & $\mathrm{E}$ & 5.3 & & 19 \\
\hline $2 / 26 / 2018$ & 23 & $\mathrm{ESE}$ & 3.8 & & 20 \\
\hline & & & & & \\
\hline
\end{tabular}

\begin{tabular}{|c|c|c|c|c|c|}
\hline \multicolumn{5}{|c|}{ Hourly weather data - Caracal meteorological station } \\
\hline & & \multicolumn{2}{|c|}{ Wind } & $\begin{array}{c}\text { Precipitation } \\
\text { for } 3 \text { hours }\end{array}$ & $\begin{array}{c}\text { Layer of } \\
\text { snow }\end{array}$ \\
\hline Date & Hour & Direction $\left(^{\circ}\right)$ & Speed $(\mathrm{m} / \mathrm{s})$ & $(\mathrm{mm})$ & $(\mathrm{cm})$ \\
\hline $2 / 27 / 2018$ & 00 & ESE & 2.2 & 2.2 & 22 \\
\hline $2 / 27 / 2018$ & 01 & E & 4.2 & & 24 \\
\hline $2 / 27 / 2018$ & 02 & E & 4.8 & & 25 \\
\hline $2 / 27 / 2018$ & 03 & ESE & 4 & 1.0 & 27 \\
\hline $2 / 27 / 2018$ & 04 & ESE & 6.5 & & 28 \\
\hline $2 / 27 / 2018$ & 05 & SE & 1.9 & & 29 \\
\hline $2 / 27 / 2018$ & 06 & SE & 1.8 & 1.5 & 31 \\
\hline $2 / 27 / 2018$ & 07 & SE & 1.5 & & 32 \\
\hline $2 / 27 / 2018$ & 08 & SE & 0.9 & & 33 \\
\hline $2 / 27 / 2018$ & 09 & SE & 1.6 & 1.6 & 33 \\
\hline $2 / 27 / 2018$ & 10 & ESE & 2.3 & & 34 \\
\hline $2 / 27 / 2018$ & 11 & ESE & 4.4 & & 35 \\
\hline $2 / 27 / 2018$ & 12 & ESE & 5.6 & 1.4 & 35 \\
\hline $2 / 27 / 2018$ & 13 & ESE & 4.4 & & 35 \\
\hline $2 / 27 / 2018$ & 14 & ESE & 4.4 & & 35 \\
\hline $2 / 27 / 2018$ & 15 & ESE & 3.5 & 0.2 & 35 \\
\hline $2 / 27 / 2018$ & 16 & E & 3.7 & & 35 \\
\hline $2 / 27 / 2018$ & 17 & ESE & 2.9 & & 34 \\
\hline $2 / 27 / 2018$ & 18 & ENE & 3.3 & & 34 \\
\hline $2 / 27 / 2018$ & 19 & ENE & 3.1 & & 34 \\
\hline $2 / 27 / 2018$ & 20 & E & 3.4 & & \\
\hline $2 / 27 / 2018$ & 21 & E & 7.7 & & \\
\hline $2 / 27 / 2018$ & 22 & E & 8.1 & & \\
\hline $2 / 27 / 2018$ & 23 & E & 6.7 & & \\
\hline
\end{tabular}

In Table 4 for Drăgășani meteorological station, shortly after begging of the blizzard the snow layer was $9 \mathrm{~cm}$ and the second day reached $29 \mathrm{~cm}$.

Table 4. Meteorological data for Drăgășani meteorological station for February $26^{\text {th }}$ and $27^{\text {th }}$ (source: Romanian National Meteorological Administration)

\begin{tabular}{|c|c|c|c|c|c|}
\hline \multicolumn{6}{|c|}{ Hourly weather data - Drăgășani meteorological station } \\
\hline & & \multicolumn{2}{|c|}{ Wind } & $\begin{array}{c}\text { Precipitation } \\
\text { for } 3 \text { hours }\end{array}$ & $\begin{array}{c}\text { Layer of } \\
\text { snow }\end{array}$ \\
\hline Date & Hour & Direction $\left(^{\circ}\right)$ & Speed $(\mathrm{m} / \mathrm{s})$ & $(\mathrm{mm})$ & $(\mathrm{cm})$ \\
\hline $2 / 26 / 2018$ & 00 & ENE & \begin{tabular}{|l|}
6.6 \\
\end{tabular} & 0.9 & 9 \\
\hline $2 / 26 / 2018$ & 01 & $\mathrm{E}$ & 6 & & \\
\hline $2 / 26 / 2018$ & 02 & $\mathrm{E}$ & 5.7 & & \\
\hline $2 / 26 / 2018$ & 03 & $\mathrm{E}$ & 6.2 & 1.0 & 10 \\
\hline $2 / 26 / 2018$ & 04 & $\mathrm{E}$ & 6.1 & & \\
\hline $2 / 26 / 2018$ & 05 & $E$ & 5.1 & & \\
\hline 2/26/2018 & 06 & $\mathrm{E}$ & 4.6 & 1.1 & 11 \\
\hline $2 / 26 / 2018$ & 07 & $\mathrm{E}$ & 4.8 & & \\
\hline $2 / 26 / 2018$ & 08 & $E$ & 5.4 & & \\
\hline $2 / 26 / 2018$ & 09 & $\mathrm{E}$ & 4.7 & 0.6 & 12 \\
\hline $2 / 26 / 2018$ & 10 & $\mathrm{E}$ & 4.2 & & 12 \\
\hline $2 / 26 / 2018$ & 11 & $\mathrm{E}$ & 5.3 & & 12 \\
\hline $2 / 26 / 2018$ & 12 & $E$ & 8.5 & 0.5 & 13 \\
\hline $2 / 26 / 2018$ & 13 & $E$ & 7.7 & & 13 \\
\hline $2 / 26 / 2018$ & 14 & $E$ & 7.9 & & 14 \\
\hline $2 / 26 / 2018$ & 15 & $E$ & 6.7 & 0.9 & 14 \\
\hline $2 / 26 / 2018$ & 16 & $E$ & 7.2 & & 14 \\
\hline $2 / 26 / 2018$ & 17 & $E$ & 6.3 & & 15 \\
\hline $2 / 26 / 2018$ & 18 & $E$ & 6.5 & 1.3 & 15 \\
\hline $2 / 26 / 2018$ & 19 & ESE & 4.8 & & 15 \\
\hline $2 / 26 / 2018$ & 20 & ESE & 5.4 & & 16 \\
\hline $2 / 26 / 2018$ & 21 & ESE & 2.6 & 1.6 & 16 \\
\hline $2 / 26 / 2018$ & 22 & ESE & 3.6 & & 16 \\
\hline $2 / 26 / 2018$ & 23 & $\mathrm{E}$ & 2.9 & & 17 \\
\hline
\end{tabular}

\begin{tabular}{|c|c|c|c|c|c|}
\hline \multicolumn{5}{|c|}{ Hourly weather data - Drăgășani meteorological station } \\
\hline & & \multicolumn{2}{|c|}{ Wind } & $\begin{array}{c}\text { Precipitation } \\
\text { for } 3 \text { hours }\end{array}$ & $\begin{array}{c}\text { Layer of } \\
\text { snow }\end{array}$ \\
\hline Date & Hour & Direction $\left(^{\circ}\right)$ & Speed $(\mathrm{m} / \mathrm{s})$ & $(\mathrm{mm})$ & $(\mathrm{cm})$ \\
\hline $2 / 27 / 2018$ & 00 & E & 4.2 & 0.9 & 17 \\
\hline $2 / 27 / 2018$ & 01 & ENE & 5.7 & & 18 \\
\hline $2 / 27 / 2018$ & 02 & E & 5.6 & & 19 \\
\hline $2 / 27 / 2018$ & 03 & E & 4.7 & 2.0 & 20 \\
\hline $2 / 27 / 2018$ & 04 & SE & 3.1 & & 20 \\
\hline $2 / 27 / 2018$ & 05 & SSE & 2.4 & & 21 \\
\hline $2 / 27 / 2018$ & 06 & S & 2.7 & 3.4 & 23 \\
\hline $2 / 27 / 2018$ & 07 & SSV & 1.7 & & 25 \\
\hline $2 / 27 / 2018$ & 08 & SSV & 2.5 & & 27 \\
\hline $2 / 27 / 2018$ & 09 & SSV & 2.6 & 2.2 & 28 \\
\hline $2 / 27 / 2018$ & 10 & SSV & 1.9 & & 28 \\
\hline $2 / 27 / 2018$ & 11 & S & 1.1 & & 29 \\
\hline $2 / 27 / 2018$ & 12 & S & 1.5 & 1.7 & 29 \\
\hline $2 / 27 / 2018$ & 13 & S & 2 & & 29 \\
\hline $2 / 27 / 2018$ & 14 & SE & 1.5 & & 28 \\
\hline $2 / 27 / 2018$ & 15 & SE & 0.9 & 0.0 & 27 \\
\hline $2 / 27 / 2018$ & 16 & S & 1.6 & & 26 \\
\hline $2 / 27 / 2018$ & 17 & SE & 1.6 & & 26 \\
\hline $2 / 27 / 2018$ & 18 & SE & 4.3 & 0.1 & 26 \\
\hline $2 / 27 / 2018$ & 19 & ESE & 2.7 & & \\
\hline $2 / 27 / 2018$ & 20 & E & 2.4 & & \\
\hline $2 / 27 / 2018$ & 21 & E & 5.2 & 0.2 & \\
\hline $2 / 27 / 2018$ & 22 & E & 6.3 & & \\
\hline $2 / 27 / 2018$ & 23 & E & 8.7 & & \\
\hline & & & & & \\
\hline
\end{tabular}


Wind direction during these two days was mostly east, east-southeast and the wind speed reached maximum in the afternoon of February $26^{\text {th }}$.

All of the information regarding the thickness of the snow layer from the tables above can be much easily observed in Fig. 6 below.

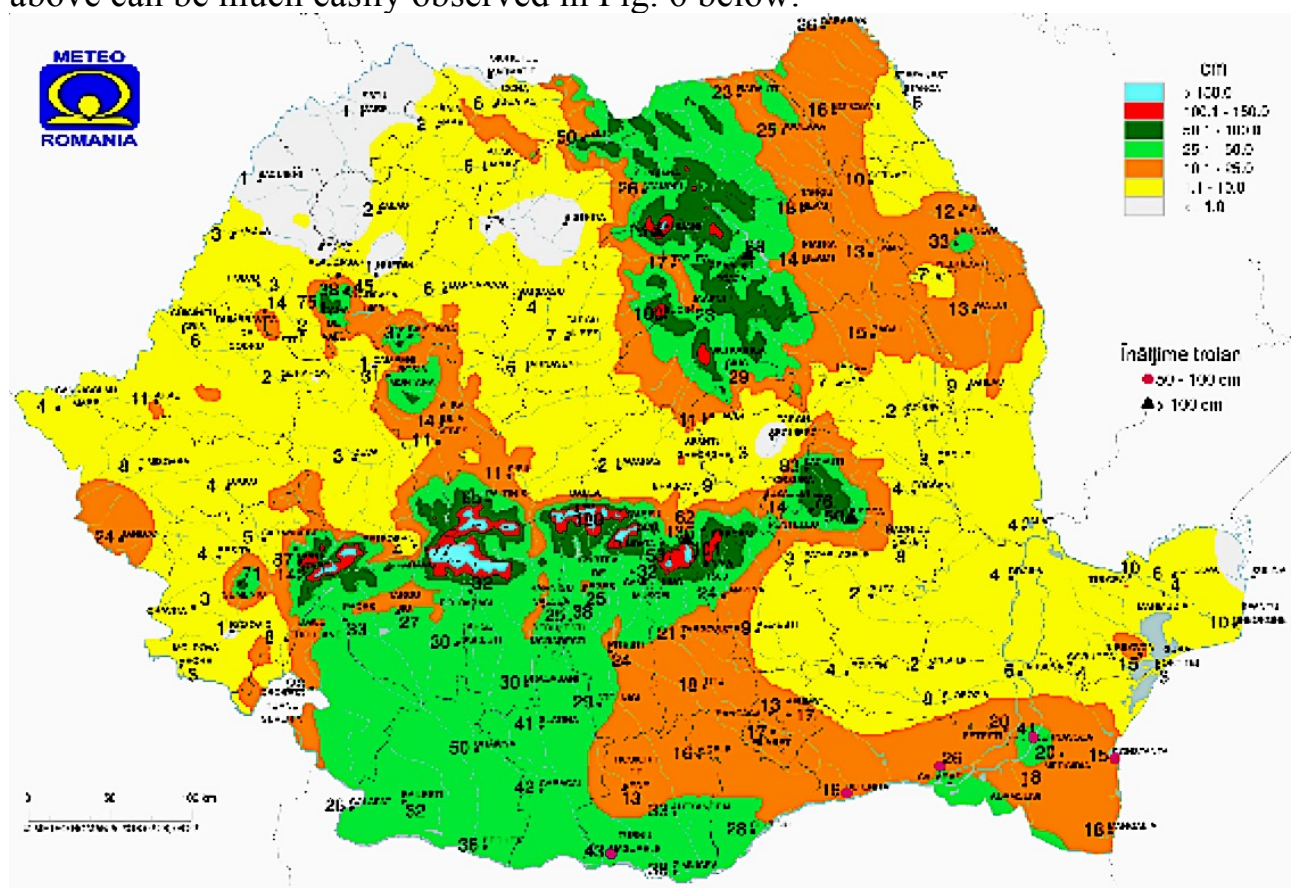

Fig. 6. Snow layer in Romania for 2018 February $28^{\text {th }}$, 06 UTC (source: Romanian National Meteorological Administration, www.meteoromania.ro)

\section{HYDROLOGICAL DATA}

Regarding the hydrological data, they come from a series of hydrometric stations, located on the rivers in the Oltenia region, namely Jiu, Olt and Danube. There were taken under analysis the followings: average and maximum daily liquid levels and flows, as well as the recorded amount of precipitation during the research time span. The eleven hydrometric stations considered are: Rovinari, Filiași, Podari, Zaval (on the Jiu River); Râmnicu Vâlcea, Oteteliș, Reșca (on the Olt river); Gruia, Calafat, Bechet, Corabia (on the Danube river).

In the graphical representations of the liquid levels (Fig. 7. and Fig. 8.) is to be observed a similar, constant, without major changes evolution for both maximum and average levels, for all eleven stations. The highest levels were recorded at the following stations on the Danube River: Gruia and Bechet, and for the Jiu and Olt Rivers: Podari and Reșca. At the opposite side, the lowest levels were at the Ostetelis station on the Olt River.

All recored levels between February $24^{\text {th }}$ and March $3^{\text {rd }}$ were lower than the established defense quotas. 


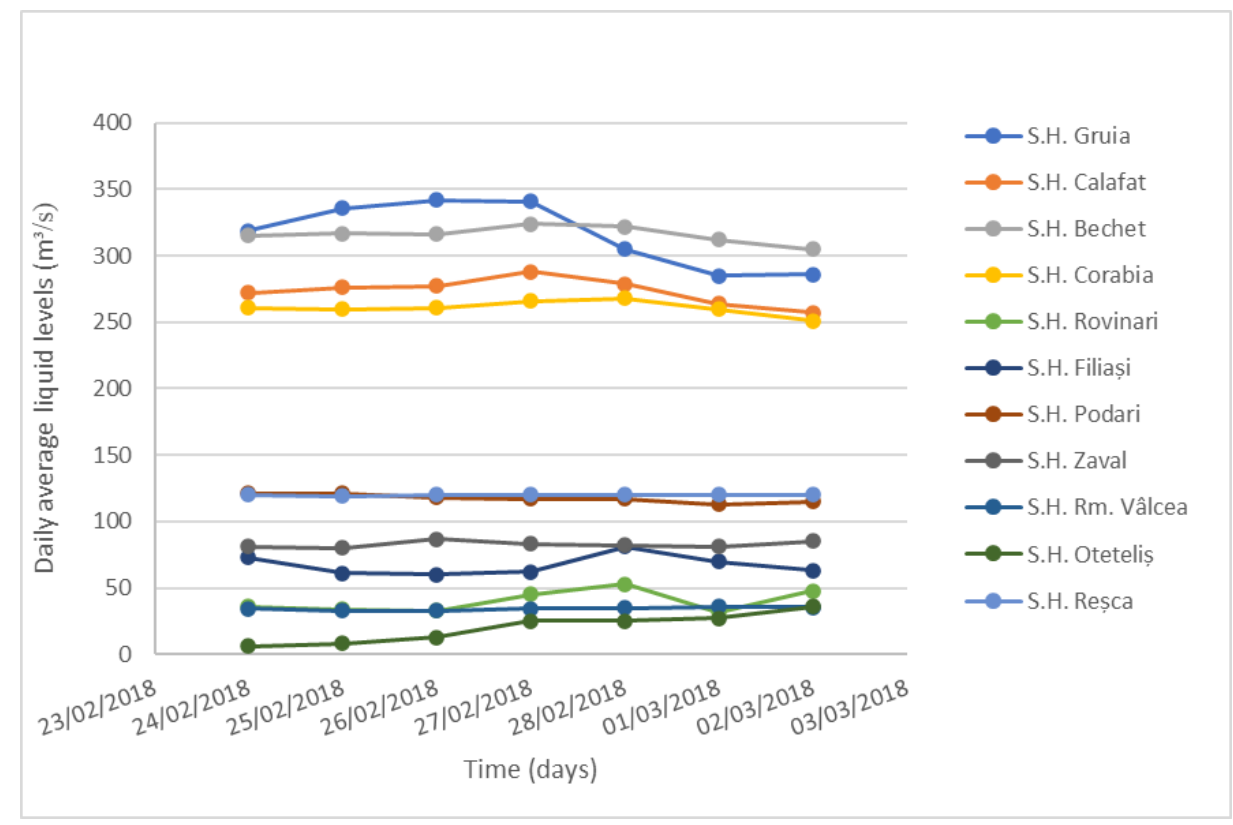

Fig. 7. Daily average liquid levels evolution in Oltenia between February $24^{\text {th }}$ and March $3^{\text {rd }}$ (source: National Institute for Hydrology and Water Management)

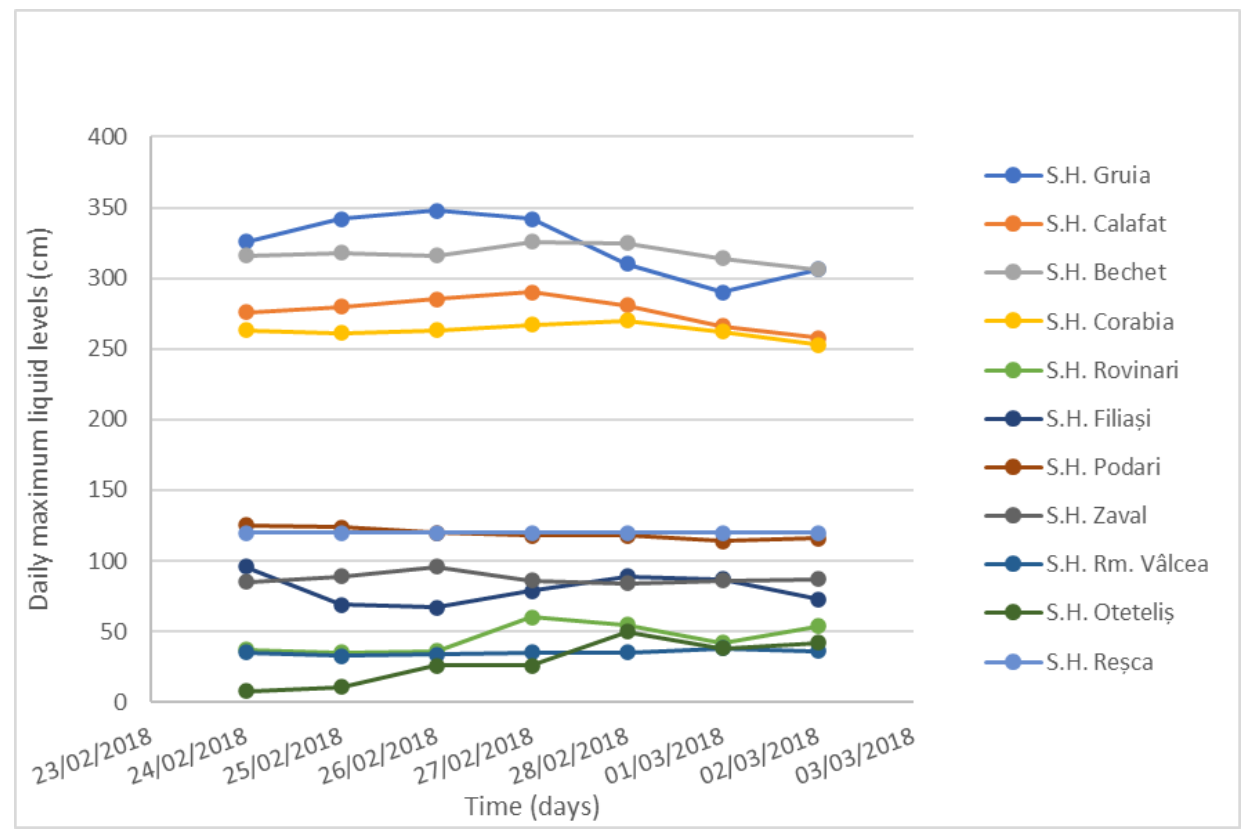

Fig. 8. Daily maximum liquid levels evolution for the hidrological stations on the Jiu, Olt and Danube Rivers in Oltenia region between February $24^{\text {th }}$ and March $3^{\text {rd }}$ (source: National Institute for Hydrology and Water Management) 
According to the evolution presented in Fig. 9. and Fig. 10. the average and maximum daily flows at the hydrometric stations in the Danube basin for Oltenia region, in the analyzed period the identified variations are relatively small. The lowest values are for Gruia and the highest ones for Bechet and Corabia with values around $6400 \mathrm{~m}^{3} / \mathrm{s}$. The greatest flows were recorded throw the middle of the time span.

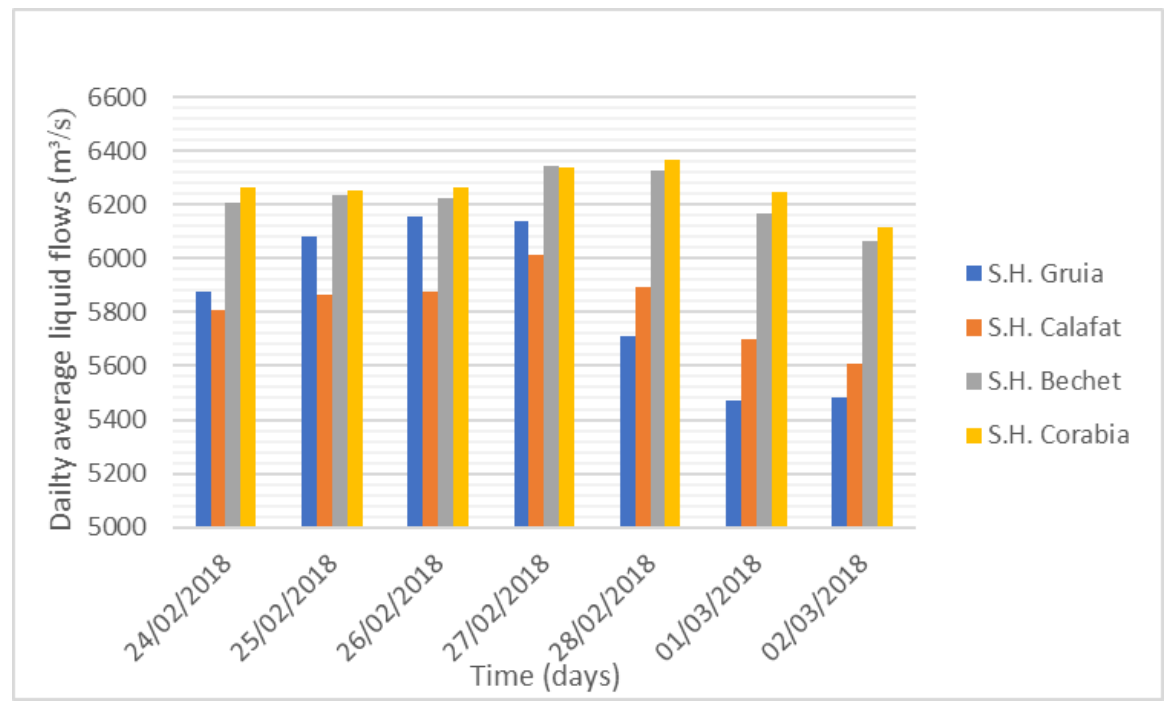

Fig. 9. Daily average liquid flows evolution for the Danube Rivers basin in Oltenia between February $24^{\text {th }}$ and March $3^{\text {rd }}$ (source: National Institute for Hydrology and Water Management)

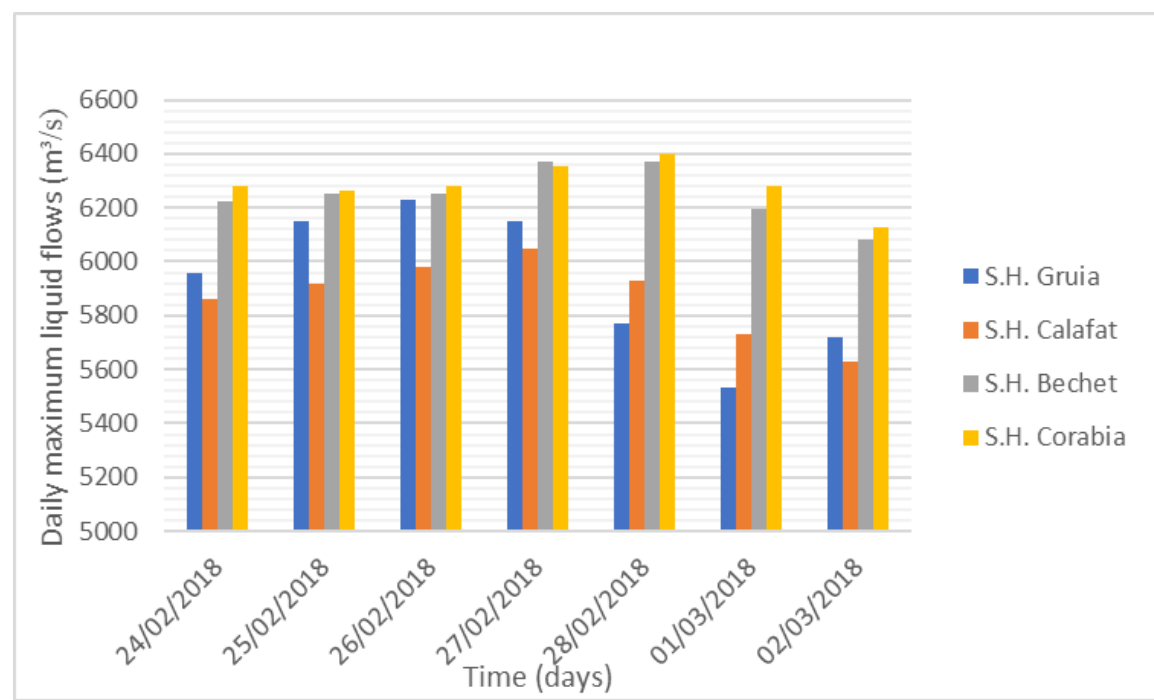

Fig. 10. Daily maximum liquid flows evolution for the Danube Rivers basin in Oltenia between February $24^{\text {th }}$ and March $3^{\text {rd }}$ (source: National Institute for Hydrology and Water Management) 
In the graphics below (Fig. 11. and Fig. 12.) among the analyzed stations, Râmnicu Vâlcea, Oteteliș and Reșca on the Olt river have recorded the lowest values for the average and maximum daily flows (below $10 \mathrm{~m}^{3} / \mathrm{s}$ ), compared to the others.

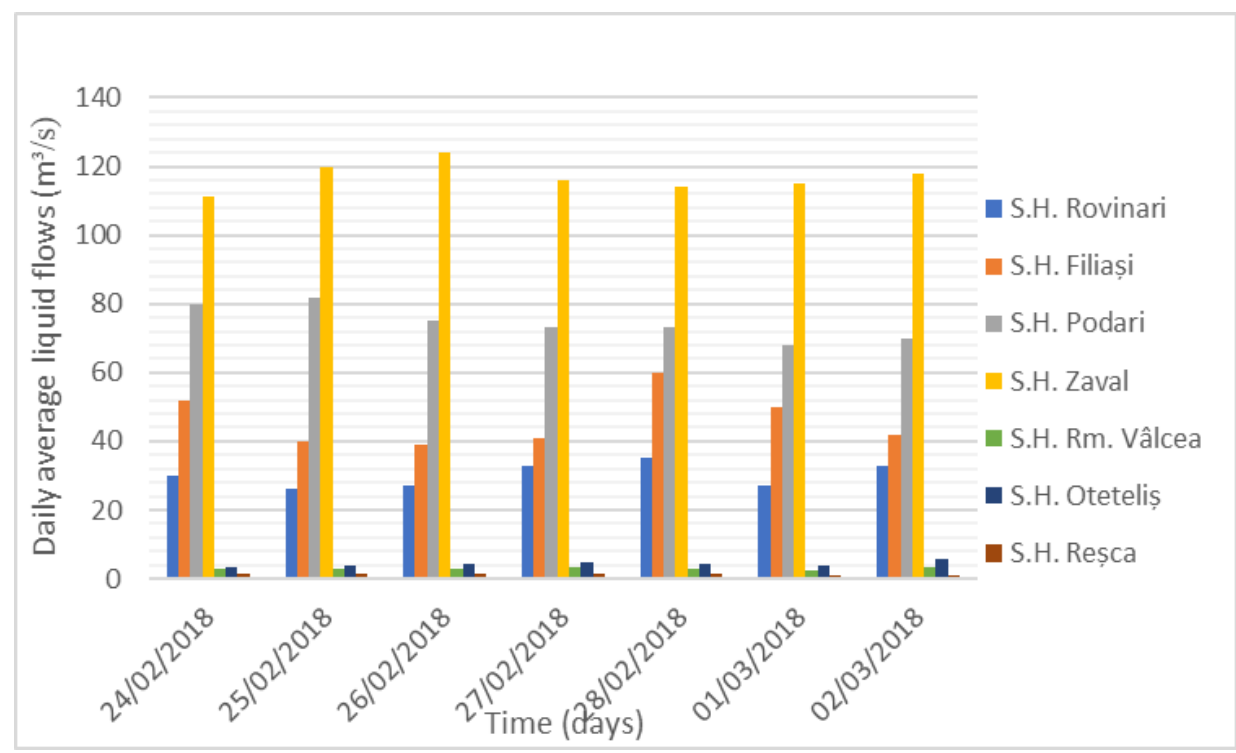

Fig. 11. Daily average liquid flows evolution for Jiu and Olt Rivers in Oltenia region between February $24^{\text {th }}$ and March $3^{\text {rd }}$ (source: National Institute for Hydrology and Water Management)

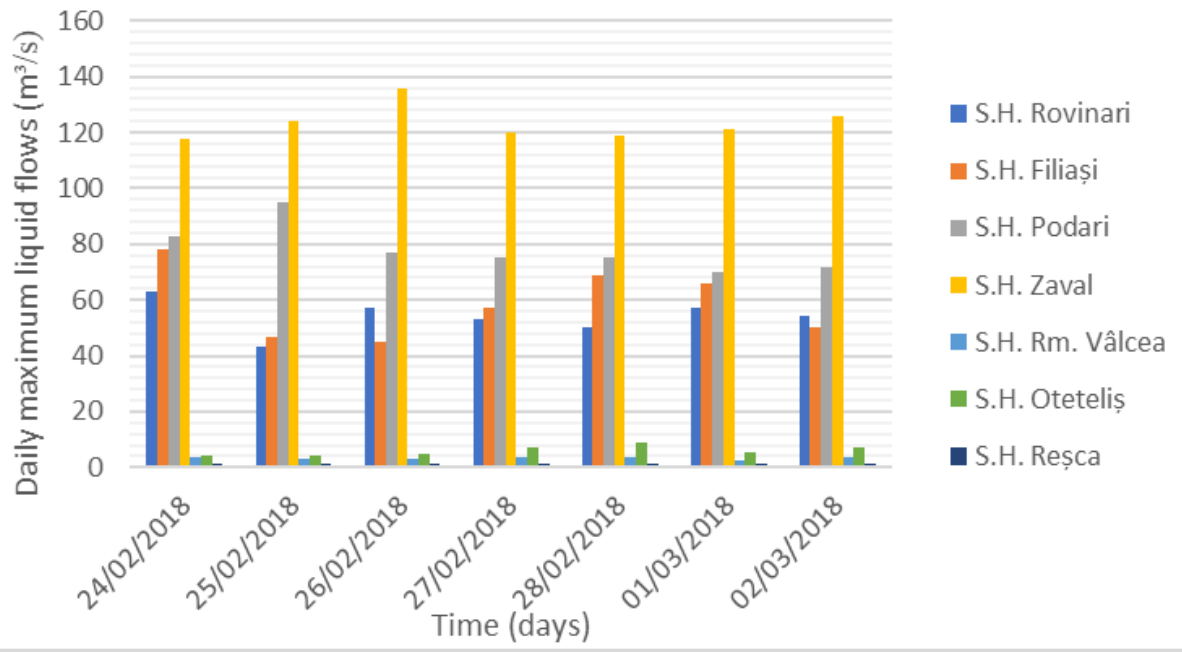

Fig. 12. Daily maximum liquid flows evolution for Jiu and Olt Rivers in Oltenia region between February $24^{\text {th }}$ and March $3^{\text {rd }}$ (source: National Institute for Hydrology and Water Management) 
On the other hand, the highest average and maximum daily flows were recorded at Zaval on the Jiu River $\left(124 \mathrm{~m}^{3} / \mathrm{s}\right.$ and $\left.136 \mathrm{~m}^{3} / \mathrm{s}\right)$, both on February $26^{\text {th }}$.

In Fig. 13. the highest value for the amount of precipitation recorded during the blizzard episode is $64 \mathrm{l} / \mathrm{m}^{2}$ at the hydrometric stations Podari on the Jiu River on February $27^{t h}$. On this day, all seven station from Jiu and Olt Rivers recorded significant amounts of precipitation. Zaval hydrometric station had a total amount of $141 \mathrm{l} / \mathrm{m}^{2}$ during the entire period. The lowest values for the amount of precipitation were recorded in the Oltenia region on February $24^{\text {th }}$ and March $1^{\text {st }}$.

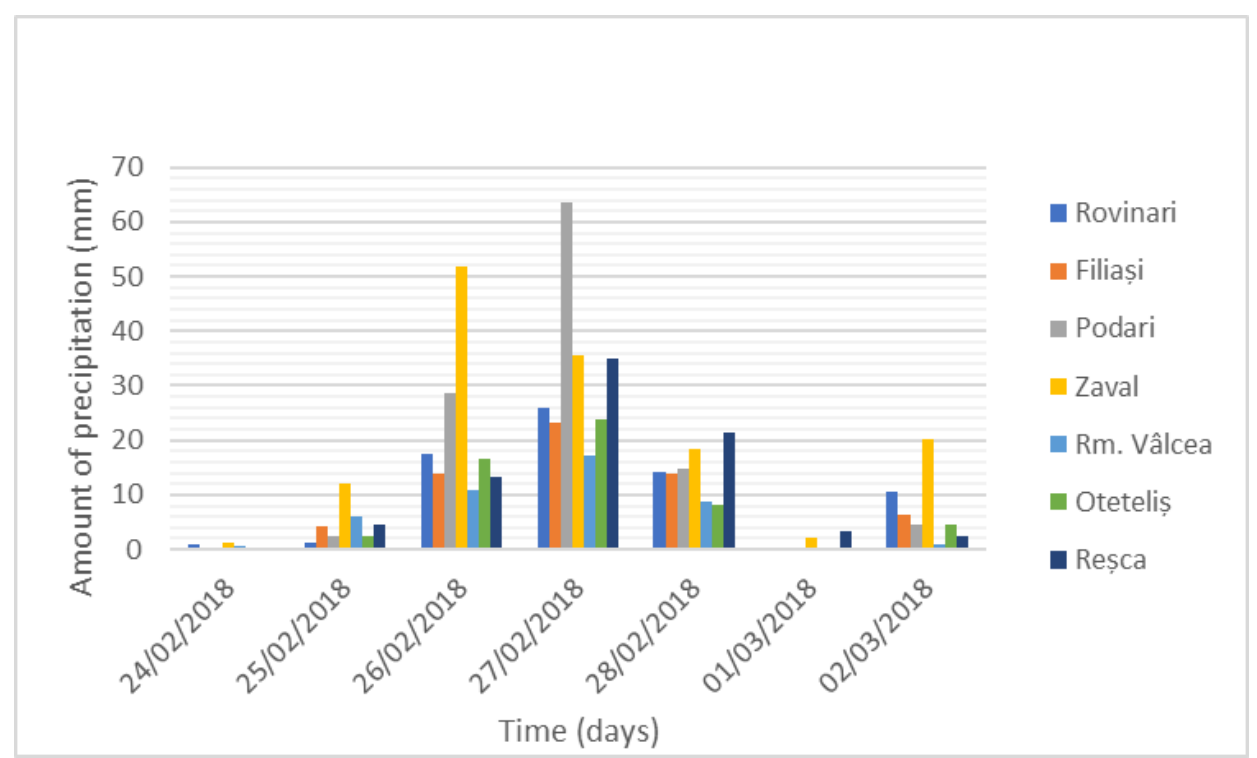

Fig. 13. Evolution of the amount of precipitation for Jiu and Olt Rivers in Oltenia region between February $24^{\text {th }}$ and March $3^{\text {rd }}$ (source: National Institute for Hydrology and Water Management)

Between February $24^{\text {th }}$ and March $3^{\text {rd }}$ for every hydrometric station located on the rivers Jiu, Olt and Danube in the Oltenia region no flood phenomena was registered. However, phenomena determined by the meteorological conditions of the blizzard were reported, such as: layer of snow with different thicknesses for all hydrometric stations, ice on the shore at Râmnicu Vâlcea and Oteteliș stations and ice on certain sectors of the river, also for Râmnicu Vâlcea station.

\section{CONCLUSIONS}

In order for a blizzard to develop in Romania a coupling mechanism is needed: a high-pressure area in the north or north-east of Romania, an anticyclone with continental polar characteristics (cold and dry) and a low-pressure area evolving over our country with warmer and humid air. 
The numerical weather forecast models captured well enough the evolution of the blizzard episode, but also the cold wave before that. Romanian National Meteorological Administration issued warnings: an orange alert for very low temperatures during both night and day and a mixed yellow (counties in the north of Oltenia and towards the end only for the southern ones) and orange (counties in the south of the region) alert for the blizzard.

The effects consisted in: a snow layer recorded both at the meteorological and hydrological stations, wind gusts and ice on the rivers.

\section{REFERENCES}

1. Bălescu O., Beşleagă N. (1962), Viscolele în R.P.R.. C.S.A., Institutul Meteorologic, Bucureşti

2. Bogdan O. et al. (2020), Anomalii climatice în Oltenia. Editura Ecran Magazin, Bucureşti

3. Bogdan O., Niculescu E. (1999), Riscurile climatice din România. Academia Română, Institutul de Geografie, Editura SEGA-INTERNAȚIONAL, Bucureşti

4. Bordei Ecaterina-Ion (1983), Rolul lanţului alpino-carpatic în evoluţia ciclonilor mediteraneeni. Editura Academiei R.S.R., Bucureşti

5. Doneaud A., Beşleagă N. (1966), Meteorologie sinoptică, dinamică şi aeronautică. Manual pentru şcoli tehnice. Ed. Didactică şi Pedagogică, Bucureşti,

6. Roșu, Al. (1973), Geografia Fizica a României. Editura didactică şi Pedagogică, București

7. Teodoreanu E. (2004), Geografie medical. Editura Academiei Române, București

8. Țîșlea D., Bacinschi D., Sârbu V., Nor R. (1965), Dicționar meteorologic. C.S.A., IMH, București

9. *** Clima Romaniei (2008), Editura Academiei Române, București

10. Online resources:

- http://wwwl.wetter3.del

- https://www.metoffice.gov.uk/

- http://aerapa.conference.ubbcluj.ro/2010/pdf/CManescu.pdf

- http://www.qreferat.com/referate/geografie/Viscolul224.php

- http://www.scritub.com/diverse/meteorologie/FENOMENE-ATMOSFERICEDE-RISC-C9412102423.php

- http://www.jurnalulolteniei.ro/2018/02/26/craiova-activitate-utilajelor-dedeszapezire-ingreunata-de-viscol/)

- http://www.gds.ro/Local/2018-02-27/primaria-craiova,-fentata-de-firmele-dedeszapezirel 